\title{
RECENT ADVANCES IN MODELING WAVE ATTENUATION BY VEGETATION
}

\author{
Qin Chen, Northeastern University, q.chen@northeastern.edu \\ Ling Zhu, Northeastern University, l.zhu@northeastern.edu \\ Agnimitro Chakrabarti, FTN Associates, achakr2@Isu.edu
}

\section{INTRODUCTION}

In the past decade, interest in wave attenuation by vegetation has increased considerably as coastal engineers and scientists search for sustainable solutions to mitigate the impacts of climate change and natural hazards. The interactions of surface weaves and natural vegetation span over a large range of scales, from turbulence and eddies at the vegetation stem scale to wave generation in vast inundated wetlands of hundreds of square miles under hurricane conditions. Restoring coastal wetlands and reducing flood risks of coastal communities require improved understanding and better predictive capability for wave attenuation over inundated coastal landscapes with vegetation. The objective of this paper is to present recent advances in multi-scale modeling of wave attenuation by wetland vegetation. Numerical modeling results ranging from vegetationresolved large eddy simulation under idealized conditions to incorporating vegetation-induced drag forces into conservation laws of momentum and energy for engineering applications will be shown. Effects of vegetation flexibility and various wave theories on the prediction of wave attenuation and the choice of vegetation drag coefficients will be discussed.

\section{METHODOLOGY}

Most of the existing models for wave-vegetation interactions utilize the Morison-type equations to parameterize the drag force acting on the flow due to the vegetation stems approximated as rigid cylinders of small diameter. An empirical drag coefficient has been used to represent the un-resolved physics in the flow resistance formula due to vegetation, which has to be calibrated for different wave models and different vegetation conditions. To gain insight into the turbulence generated by vegetation, Chakrabarti et al. (2016) conduct a large eddy simulation (LES) of waves over rigid vegetation using OpenFOAM. They compute the drag coefficient from the forces acting on each stem. Although both LES-based and energy-based models were tested against laboratory data with good agreement, it is only applicable to rigid vegetation. Zhu and Chen (2015) developed a phaseresolving, momentum-based model that couples waves with flexible vegetation. The vegetation model solves the complete force balance equation for beam deformation using a high-order finite-element method. During a hurricane event, salt marshes remain emergent at the beginning and ending of the water surge while become completely submerged at the peak of the surge. For surface waves over flooded wetlands, the drag coefficient strongly depends on wave models, vegetation properties (vegetation spacing, stem diameter, plant height and flexural rigidity, etc.) as well as the flow depth and speed (Zhao and Chen 2016, Zhu an Chen 2017, Chakrabarti et al. 2017).

\section{RESULTS}

The study utilizes a phase-averaged energy-based model, and phase-resolving Boussinesq, Euler and Navier-Stokes (N-S) solvers with different representations of submerged vegetation. Fig. 1 (left) shows the effect of the vegetation flexibility on wave attenuation. By resolving each vegetation stem using the large eddy simulation technique, the vegetation drag force can be obtained directly from the N-S model without prescribing a drag coefficient and using the Morison-type equations. Fig 1 (right) illustrates the modeled vorticity field in a submerged canopy under the wave action.

\section{CONCLUSIONS}

Using the large eddy simulation approach, we gain insight into the stem-scale vorticity dynamics in a canopy without invoking the Morison-type parameterization of vegetation drag. Using an Euler solver coupled with a deformable vegetation model, we obtain a better understanding of the effect of plant flexibility on the reduction of wave attenuation. By connecting the small-scale momentumbased models with the large-scale energy-based model, we are able to explain the spectral-varying drag coefficients inferred from the field observations. The validated numerical models will help mitigate the negative impact of hurricanes on coastal communities.


Figure 1- Modeled wave attenuation by flexible vegetation (left), and stem-scale vorticity of rigid vegetation (right).

\section{REFERENCES}

Chakrabarti, A., Chen, Q., Smith, H. and Liu, D. (2016): Large eddy simulation of unidirectional and wave flows through vegetation, J. Eng. Mech. 142 (8): 04016048.

Chakrabarti, A., Brandt, S. R., Chen, Q. \& Shi, F. (2017): Boussinesq modeling of wave-induced hydrodynamics in coastal wetlands. J. Geo. Res., 122: 3861-3883.

Zhu, L., and Chen, Q. (2015): Numerical modeling of surface waves over submerged flexible vegetation. J. Eng. Mech., 141 (8): A4015001.

Zhao, H. and Chen, Q. (2016): Modeling attenuation of storm surge over deformable vegetation: Parametric study. J. Eng. Mech., 142 (8): 06016006.

Zhu, L. \& Chen, Q. (2017): Modeling attenuation of highly nonlinear water waves by rigid vegetation: A comparison of different wave theories, J. WPCOE, 143 (5): 04017029. 